\title{
Genetic Variants in Nicotinamide-N-Methyltransferase (NNMT) Gene are Related to the Stage of Non-Alcoholic Fatty Liver Disease Diagnosed by Controlled Attenuation Parameter (CAP)-FibroScan
}

\author{
Eman M. Hasan', Rasha A. Abd Al Aziz ${ }^{1}$, Dina Sabry², Samar K. Darweesh ${ }^{1}$, Hedy A. Badary ${ }^{1}$, Aisha Elsharkawy ${ }^{1}$, \\ Mahmood M. Abouelkhair ${ }^{1}$, Ayman Yosry ${ }^{1}$
}

1) Hepato-Gastroenterology

and Endemic Medicine

Department,

2) Medical Biochemistry

Department,

Faculty of Medicine,

Cairo University

Cairo, Egypt

\begin{abstract}
Address for correspondence: Dr. Samar Kamal Darweesh Associate Professor of Hepatogastroenterology and Tropical Medicine Department, Faculty of Medicine, Cairo University, Cairo, Egypt samarkad@hotmail.com
\end{abstract}

\begin{abstract}
Background \& Aims: Various genetic polymorphisms play a key-role in the pathogenesis of NAFLD and progression to NASH with fibrosis to cirrhosis. We aimed to study the association between single-nucleotide polymorphisms (SNPs) in NNMT gene, namely rs694539 and the development of different stages of NAFLD diagnosed by controlled attenuation parameter (CAP) of FibroScan Echosens ${ }^{\oplus}$.

Methods: Transient elastography (FibroScan ${ }^{\circledast}$ ) with controlled attenuation parameter (CAP) measurement was performed in 81 NAFLD patients ( 35 of them with liver biopsy) and 80 non-NAFLD controls. The accuracy of CAP and FibroScan for the detection and quantification of hepatic steatosis/fibrosis, respectively, was assessed based on liver biopsy aspect. Genetic variants of NNMT gene rs694539 were analyzed using a polymerase chain reaction-restriction fragment length polymorphism (PCR-RFLP).

Results: According to BMI (kg/m²), among the patients, $17(21 \%)$ were overweight, $56(69.1 \%)$ obese, and $8(9.9 \%)$ morbidly obese. CAP and FibroScan diagnosed steatosis/fibrosis correlated significantly with liver biopsy. There was a significant association between polymorphisms of rs694539-NNMT gene and NAFLD presence and stages. The mutant type (AA-genotype) was found in 33\% NAFLD patients versus $1.2 \%$ controls $(\mathrm{P}<0.001)$, whereas the wild type (GG-genotype) was present in $21 \%$ versus $63.8 \%$ controls $(\mathrm{P}<0.001)$. Moreover, the AA-genotype significantly correlated with the steatosis degree by CAP but not the fibrosis degree by FibroScan. Multivariate regression analysis of all the independent risk factors showed non-significant correlations with the degree of steatosis on CAP. However, by using a stepwise approach, waist circumference showed significance as an independent predictor of NAFLD.

Conclusions: Polymorphisms in rs694539-NNMT gene (mutant AA-genotype) could be a genetic risk factor for developing NAFLD and NASH (indicating susceptibility for progression and complications). Individuals with wild type (GG-genotype) are at less risk of NAFLD development. CAP and FibroScan efficiently diagnosed steatosis and fibrosis.
\end{abstract}

Key words: Non-alcoholic Fatty Liver Disease (NAFLD) - Non-alcoholic Steatohepatitis (NASH) Nicotinamide-N-methyltransferase (NNMT) gene - Single Nucleotide Polymorphisms (SNPs) - Controlled attenuation parameter (CAP) - FibroScan.

Abbreviations: CAP: controlled attenuation parameter; FBG: fasting blood glucose; LSM: Liver stiffness measurement; NAFLD: Non-alcoholic fatty liver disease; NASH: Non-alcoholic Steatohepatitis; NNMT: Nicotinamide-N-methyltransferase; PCR-RFLP: Polymerase chain reaction-Restriction fragment length polymorphism; SNPs: Single Nucleotide Polymorphisms; TE: transient elastography; TG: triglycerides; T2DM: type 2 dabetes mellitus.

\section{INTRODUCTION}

Non-alcoholic fatty liver disease (NAFLD) is the hepatic manifestation of obesity and is rapidly becoming the most common liver disease worldwide with a prevalence of $21.3 \%$. About $2-3 \%$ of the general population is estimated to have non-alcoholic steatohepatitis (NASH), which may progress to liver cirrhosis and hepatocellular carcinoma (HCC) [1].

NAFLD prognosis is predominantly determined by the liver fibrosis degree and steatohepatitis. This emphasizes the need for accurate differentiation between NASH and NAFL to determine prognosis and optimal treatment [2]. 
NAFLD develops from a complex process including genetic susceptibility and environmental insults. Susceptibility to highprevalence diseases such as obesity, type 2 diabetes mellitus (T2DM), cardiovascular disease, and NAFLD comprises a heritable component variously accounting for up to 30 to $50 \%$ of the relative risk [3].

Genome-wide association studies identified patatin-like phospholipase domain-containing 3 (PNPLA3) I148M gene and transmembrane 6 superfamily member 2 (TM6SF2) E167K gene variants, as major determinants of liver steatosis and susceptibility to progressive NASH. Both can determine liver fat accumulation through lipid droplets and very lowdensity lipoprotein (VLDL) modifications. Several other genetic variants, including rare mutations, involved in the regulation of hepatocellular lipid metabolism, are being scrutinized [4].

The possible role of nicotinamide- $\mathrm{N}$-methyltransferase (NNMT) gene rs694539 variant in the development of NASH was strongly associated with increased risk for NASH. Individuals with the GG genotype appeared to have protection against $\mathrm{NASH}$, whereas the AA genotype showed statistically significant increased risk for NASH [5].

The prognosis and outcome of the patients with NAFLD is predominantly determined by the degree of liver fibrosis and steatohepatitis. This emphasizes the need for accurate differentiation between NASH and NAFLD in order to determine the prognosis and optimal treatment [2]. Currently, the routinely used modalities (laboratory tests and ultrasonography) cannot adequately determine the degrees of steatosis and fibrosis or cannot be applied as a screening procedure.

Among the non-invasive tests, transient elastography (TE) (FibroScan ${ }^{\circ}$ ) with controlled attenuation parameter (CAP) has demonstrated a good accuracy in quantifying the levels of liver steatosis and fibrosis in patients with NAFLD. CAP is measured using FibroScan $\left(\right.$ Echosens $s^{\circ}$ ) based on vibration-controlled transient elastography (VCTE), a technique initially developed to assess liver stiffness (LS) which correlates with fibrosis. CAP measures ultrasound (US) attenuation at $3.5-\mathrm{MHz}$ using the postulate that fat affects US propagation [6].

On the other hand, the study of genetic factors in NAFLD is a rapidly growing field. To-date, the PNPLA3-I148M and TM6SF2-E167K gene variants are the major determinants of inter-individual differences in liver steatosis and susceptibility to progressive NASH. Both of these genes determine liver fat retention through lipid droplets and VLDL modifications. PNPLA3 affects directly hepatic stellate cells and retinol metabolism. These findings suggest that hepatocellular accumulation of neutral lipids is harmful. Several other genetic variants, including rare mutations, involved in the regulation of hepatocellular lipid metabolism, are being scrutinized [4].

The primary objective of this work was to evaluate the possible role of NNMT rs694539 genetic polymorphism as a risk factor for the development of NAFLD and its relation to different stages of steatosis (diagnosed by CAP and FibroScan measurement) among a cohort of patients with NAFLD.

\section{PATIENTS AND METHODS}

\section{Study population}

This cross-sectional study included 81 patients attending the Hepatology outpatient clinic and Cairo University Centre for Hepatic fibrosis (CUC-HF) of Kasr Al-Ainy Hospital, Faculty of Medicine, Cairo University over a period of 12 months (2/2015 to 2/2016), who had NAFLD. In addition, 80 age-matched healthy persons served as a control group. All included patients and controls are Egyptians with North African ethnicity.

\section{Inclusion criteria}

Patients with a body mass index (BMI) higher than 25.0 $\mathrm{kg} / \mathrm{m}^{2}$ were suspected to have NAFLD on basis of increased hepatic brightness by abdominal ultrasonography with exclusion of other causes of secondary steatosis: negative viral markers (HCV-Ab, HBsAg, HBc-total), negative Anti-nuclear antibody (ANA), normal serum ferritin and ceruloplasmin, negative history for significant alcohol consumption $(>20 \mathrm{~g} / \mathrm{d}$ for females and $30 \mathrm{~g} / \mathrm{d}$ for males) and for use of medications that can cause fatty liver.

Controls were selected from healthy persons with negative viral markers, normal blood tests [liver function tests, INR, CBC, fasting blood glucose (FBG), glycated hemoglobin ( $\mathrm{HbAlC})$, kidney function tests], normal BMI and normal liver appearance by ultrasonography.

\section{Patients' eligibility}

We excluded patients who were younger than 18 years old, those with decompensated cirrhosis, those having other metabolic disorders than DM, obesity and/or dyslipidemia, those with autoimmune disorders, viral hepatitis (positive HCVAb, HBsAg or HBcAb total) or current or past alcohol or hepatotoxic drugs consumption, and those with contraindication(s) for liver biopsy.

This study was approved by the department committee (IRB) and the institution Ethics Committee based on the 1975 (as revised in 1983) Declaration of Helsinki. Informed consent was obtained from all participants.

\section{Study course}

The patients were subjected to: history taking with emphasis on hepatotoxic drugs, BMI calculation, waist circumference (WC), serum ALT, AST, GGT, ALP, albumin, serum ferritin and ceruloplasmin, serum total cholesterol, HDL-cholesterol, LDL-cholesterol, triglycerides (TGs), FBG, HBsAg, HBcAb total, HCV Ab, ANA, abdominal ultrasonography especially grade of liver brightness.

CAP was performed in all patients and controls using the 3.5-MHz M-probe of Echosens Fibroscan ${ }^{\circ}$. Both TE and CAP were done at Cairo University Centre for Hepatic fibrosis (CUC-HF).

Polymerase chain reaction-Restriction fragment length polymorphism (PCR-RFLP) was applied to detect the distribution of genetic polymorphisms of the rs694539 NNMT gene in blood regarding the wild type (G-genotype) and the mutant type (A-genotype). 
Percutaneous ultrasound-guided liver biopsy was performed in 35 out of 81 patients. Two experienced hepatic pathologists blinded to the clinical data independently reviewed the histological findings. Fibrosis staging was evaluated (separately from NASH) from 0 to 4 scales. The hepatic steatosis was graded from 0 to 3 based on the number of hepatocytes with steatosis in the biopsy: S0: $<5 \%$; S1: 5-33 $\%$; S2:34-66 \%; S3:>66 \% [7]. In our study, NASH was defined as NAS score $\geq 5$ in liver biopsy. FibroScan and CAP were evaluated in reference to liver biopsy and data showed that fibrosis detected by FibroScan and steatosis detected by CAP significantly correlated to the liver biopsy findings.

Liver biopsy was performed in 35 patients only due to many reasons, since we have compared biopsy findings with FibroScan and CAP and found significant match in the fibrosis and steatosis stages between biopsy and FibroScan and CAP, respectively (results in publication). In addition, owing to the risks associated with the more invasive liver biopsy and also that some of the patients did not give consent for biopsy, we were satisfied with the CAP and FibroScan findings and decided to assess the degree of steatosis and fibrosis using only non-invasive techniques in the rest of the patients.

\section{Measurement of CAP}

All TE and CAP measurements were done as per-themanufacturer's recommendations by experienced operators blinded to the patients' clinical and histological data.

Assisted by the US image, a portion of the liver at least $6 \mathrm{~cm}$ thick and free of large vessels was identified using a portable 3.5-MHz M-probe of Echosens FibroScan ${ }^{\circ}$, which measured the liver stiffness $25-65 \mathrm{~mm}$ underneath the skin surface. The $3.5-\mathrm{MHz}$ ultrasound transducer mounted on the axis of an electromechanical transducer transmitted a vibration of $50-\mathrm{Hz}$ and induced an elastic shear wave that propagates through the underlying tissues.

The LS measurement (LSM) was expressed as $\mathrm{kPa}$, and the examination was considered reliable if 10 valid measurements were obtained, the success rate exceeded $60 \%$, and the ratio of the interquartile range (IQR) to the median of 10 measurements (IQR/M) was $<30 \%$. For this study, only LSMs using the $\mathrm{M}$-probe were considered because the CAP algorithm is specific to this device. TE cutoffs used were those previously discussed [8]. CAP is a proprietary algorithm performed simultaneously with LSM using the US signals acquired by the FibroScan to detect hepatic steatosis. CAP measures the ultrasound attenuation using signals acquired by the $3.5-\mathrm{MHz}$ FibroScan probe.

The CAP is measured only on validated measurements according to the same criteria used for LSM and on the same signals, ensuring that one obtains a liver ultrasonic attenuation simultaneously and in the same volume of liver parenchyma as LSM. The final CAP value, which ranges from 100 to 400 decibels per meter $(\mathrm{dB} / \mathrm{m})$, is the median of individual measurements. As an indicator of variability, the ratio of the IQR of CAP values to the median (IQR/MCAP) was calculated.

The steatosis cutoffs diagnosed by CAP are those of de Lédinghen et al., considering $\mathrm{S} 0=180-217, \mathrm{~S} 1=223-268$, $\mathrm{S} 2=268-301$, and $\mathrm{S} 3=301-346$ [9].

\section{RFLP-PCR procedure for NNMT polymorphism diagnosis} DNA extraction

Total DNA was isolated from whole blood mononuclear cells using the Qiagene cells/tissue extraction kit (Qiagene, USA) according to instructions of manufacturer.

The isolation of intact DNA requires four essential steps: effective disruption of cells, denaturation of nucleoprotein complexes, inactivation of endogenous deoxyribonuclease (DNase) activity and removal of contaminating RNA and proteins.

The Total DNA Isolation System combines the disruptive and protective properties of guanidine thiocyanate (GTC) and $\beta$-Mercaptoethanol to inactivate the deoxyribonucleases present in cell extracts. GTC acts to disrupt nucleoprotein complexes, allowing the DNA to be released into solution and isolated free of protein.

\section{Lysis of cells}

$175 \mu \mathrm{l}$ of AVL lysis buffer, $25 \mu \mathrm{l}$ of protease and $4 \mu \mathrm{l}$ Rase were added to the cells, the mixture was dispersed and mixed by pipetting. The mixture was incubated for 60 minutes at $55^{\circ} \mathrm{C}$.

\section{DNA purification}

$200 \mu \mathrm{l}$ of $95 \%$ ethanol was added to the cleared lysate, mixed in a fresh microcentrifuge tube then transferred to the Spin Column Assembly and centrifuged at $8000 \mathrm{rpm}$ for one minute.

$600 \mu \mathrm{l}$ of AW1 DNA wash solution was added and centrifuged at $8000 \mathrm{rpm}$ for one minute. Then, the addition of $600 \mu$ l of AW2 DNA wash solution with ethanol $(100 \mathrm{ml}$ of $95 \%$ ethanol to a bottle containing $58.8 \mathrm{ml}$ concentrated AW2 DNA wash solution) which was centrifuged at $8000 \mathrm{rpm}$ one minute. The collection tube was emptied and $250 \mu \mathrm{l}$ of AW2 DNA wash solution with ethanol were added and centrifuged at $14,000 \mathrm{rpm}$ for 2 minutes.

The spin basket was transferred from the collection tube to the elution tube, $100 \mu \mathrm{l}$ of nuclease-free water were added to the membrane and centrifuged at 12,000-14,000 rpm for one minute. The spin basket was discarded and the elution tube containing the purified DNA obtained.

The purity (A260/A280 ratio) and the concentration of DNA were obtained using spectrophotometry (dual wave length Beckman, Spectrophotometer, USA). The extracted and purified DNA samples were stored at $-80^{\circ} \mathrm{C}$ for further use.

\section{RFLP-PCR}

NNMT gene polymorphism (rs694539) was detected by RFLP-PCR method. A 187 base-pair (bp) product was obtained. EzWayTM Direct Taq PCR Master mix (Koma Biotech Inc., Seoul, Korea) in $25 \mu \mathrm{L}$ reaction volume was used. The amplification was carried out in a PCR thermal cycler Biosmetra, Germany. The PCR products were separated by $1.5 \%$ agarose gel electrophoresis and stained with ethidium bromide $(0.5 \mathrm{mg} / \mathrm{L})$. Ten $\mu \mathrm{l}$ of the PCR products were digested with 1 unit of the NlaIII restriction endonuclease (New England Biolabs, Hitchin, UK) in a total volume of $20 \mu \mathrm{l}$ at $37^{\circ} \mathrm{C}$ overnight.

Applied biosystems reagents in reaction volume conducted in the RFLP-PCR analyses using PCR Master Mix were performed in duplicate (all steps) to verify the accuracy of the detected genotypes. Both homozygous (GG and AA) and 
heterozygous genotypes (GA) were estimated on $4 \%$ agarose gel. The primers for the studied gene [5]:

Forward primer sequence $\left(5^{c}-3^{c}\right)$

ACGTTGGATGGTCCTAGAGTCCTA

Reverse primer sequence $\left(5^{\prime}-3^{\prime}\right)$

\section{ACGTTGGATGCAGCCATCTCAAATG}

\section{Statistical analysis}

Data were coded and entered using the statistical package SPSS version 22. Data was summarized using mean and standard deviation (SD) for quantitative variables and frequencies (numbers) and relative frequencies (percentages) for categorical variables. unpaired $t$ test was used when comparing two groups and analysis of variance (ANOVA) with multiple comparisons post hoc test when comparing more than two groups.

For comparing categorical data, the chi square $\left(\chi^{2}\right)$ test was performed. Exact test was used instead when the expected frequency was less than 5 . Correlations between quantitative variables were made using Spearman correlation coefficient. Genotype and allele frequencies were compared between the disease and the control groups using $\chi^{2}$ tests. Odds ratio (OR) with $95 \%$ confidence intervals was calculated. P-values less than 0.05 were considered as statistically significant.

The study of NNMT genetic SNPs in NAFLD had been performed before in only one research with a sample size of 80 patients, so we used the same sample size estimated before (81 patients in our study).

Tests of normality of distribution of genetics (Hardy Weinberg) in controls (80 samples counted) were done, where $\chi^{2}=1.77$, for likelihoods of calculated $\chi^{2}$ value (Table I), the $\mathrm{p}$ allele frequency $=0.81$; the $\mathrm{q}$ allele frequency $=$ 0.19 , the $\mathrm{P}$ value was $>0.05$ so it was not deviated from equilibrium [10].

Table I. Likelihood of calculated $\chi^{2}$ value in the test of normality of distribution of genetics in controls

\begin{tabular}{lcc}
\hline Genotype & Expected & Observed \\
\hline Common & 52.81 & 51 \\
homozygotes & & \\
Heterozygotes & 24.38 & 28 \\
Rare homozygotes & 2.81 & 1 \\
\hline
\end{tabular}

\section{RESULTS}

\section{Basic characteristics}

Among patients, ages ranged from 19 to 66 years (44.58 \pm 9.44$)$, with $49(60.5 \%)$ females and 32 (39.5\%) males. BMI ranged from $27.8 \mathrm{~kg} / \mathrm{m}^{2}$ to $46.9 \mathrm{~kg} / \mathrm{m}^{2}(33.69 \pm 4.45)$, whereas the WC was $108.33 \pm 11.29 \mathrm{~cm}$.

In the control group, ages ranged from 21 to 63 years (38.84 \pm 8.19$)$, with $48(60 \%)$ females and $32(40 \%)$ males, BMI was $25.68 \pm 1.48 \mathrm{~kg} / \mathrm{m}^{2}$, WC was $81.31 \pm 5.49 \mathrm{~cm}$.

According to BMI $\left(\mathrm{kg} / \mathrm{m}^{2}\right)$, patients and controls were categorized into normal (18.5-24.9), overweight (25.0-29.9), obese (30.0-40.0) and morbidly obese (>40.0).

Among patients, $21 \%$ (17 patients) were overweight, $69.1 \%$ (56 patients) were obese, and 9.9\% (8 patients) were morbidly obese, whereas among controls, $36.2 \%$ (29 subjects) had normal BMI, $63.8 \%$ (51 subjects) were overweight, and none were obese.

\section{Distribution of risk factors for NAFLD among patients and controls}

The known risk factors for the development of NAFLD as obesity, DM, hyperlipidemia and metabolic syndrome (MS) showed significant difference between patients and controls (Table II). All patients had negative history of alcohol or drug abuse. ALT, AST and GGT were significantly higher in patients than controls. All patients and controls had normal INR and renal function (Table II).

Table II. Distribution of clinical risks and laboratory findings among NAFLD patients and controls

\begin{tabular}{|c|c|c|c|c|c|}
\hline \multirow[t]{2}{*}{ Item } & \multicolumn{2}{|c|}{ Patients } & \multicolumn{2}{|c|}{ Controls } & \multirow[t]{2}{*}{$P$ value } \\
\hline & Number & $\%$ & Number & $\%$ & \\
\hline \multicolumn{6}{|l|}{ BMI } \\
\hline Overweight & 17 & 21 & 51 & 63.8 & $<0.001$ \\
\hline$\geq$ Obese & 64 & 79 & -- & -- & \\
\hline T2DM & 22 & 27.2 & 2 & 2.5 & $<0.001$ \\
\hline HTN & 13 & 16.0 & 0 & 0.0 & ----- \\
\hline Family history & 26 & 38.8 & 0 & 0.0 & ----- \\
\hline MS & 12 & 14.8 & 0 & 0.0 & $<0.001$ \\
\hline Smoking & 14 & 17.3 & 9 & 11.2 & 0.317 \\
\hline \multicolumn{6}{|c|}{ Laboratory findings (mean, SD) } \\
\hline T. Cholesterol & 221.94 & 58.78 & 130.22 & 27.84 & $<0.001$ \\
\hline HDL-C & 45.02 & 20.33 & 64.39 & 3.73 & $<0.001$ \\
\hline LDL-C & 135.69 & 54.88 & 91.56 & 16.07 & $<0.001$ \\
\hline TGs & 194.15 & 96.61 & 85.89 & 17.46 & $<0.001$ \\
\hline FBS & 104.30 & 38.58 & 84.84 & 9.24 & $<0.001$ \\
\hline PP-BG & 176.16 & 48.62 & 150.19 & 19.47 & $<0.001$ \\
\hline HGB & 13.12 & 1.87 & 13.86 & 1.52 & 0.505 \\
\hline PLT & 277.47 & 70.77 & 337.58 & 67.19 & 0.317 \\
\hline WBCs & 6.10 & 2.07 & 6.04 & 1.94 & 0.611 \\
\hline T. Bilirubin & 0.74 & 0.28 & 0.62 & 0.23 & 0.324 \\
\hline T. Protein & 7.12 & 0.52 & 8.04 & 0.44 & $<0.001$ \\
\hline ALB & 4.26 & 0.45 & 4.43 & 0.52 & 0.451 \\
\hline AST & 46.67 & 40.14 & 18.19 & 4.94 & $<0.001$ \\
\hline ALT & 50.02 & 35.09 & 16.08 & 5.81 & $<0.001$ \\
\hline GGT & 35.89 & 69.05 & 17.79 & 5.36 & 0.021 \\
\hline ALP & 48.59 & 21.24 & 48.42 & 22.11 & 0.505 \\
\hline INR & 1.02 & 0.08 & 1.02 & 0.05 & 0.6 \\
\hline
\end{tabular}

BMI: Body mass index; HDL-c: High Density Lipoprotein Cholesterol; HTN: hypertension; LDL-c: Low Density Lipoprotein Cholesterol; MS: metabolic syndrome; TGs: Triglycerides; FBS: Fasting Blood Sugar; PP-BG: Postprandial Blood Glucose; WBCs: White Blood Cells.

All controls showed normal hepatic echogenicity by US (mostly excluding NAFLD presence in controls). Among patients, liver brightness grade I was diagnosed in $22(27.2 \%)$, grade II in $40(49.4 \%)$ and grade III in $19(23.5 \%)$ patients.

The degree of steatosis using CAP was mild (S1) in $28.4 \%$ (23 patients), moderate (S2) in 23.5\% (19 patients) and severe in $48.1 \%$ (39 patients), whereas the degree of fibrosis using 
Table III. Correlation between degree (P1) and presence (P2, CC) of steatosis by CAP and various parameters

\begin{tabular}{|c|c|c|c|c|c|c|c|c|c|}
\hline \multirow[t]{2}{*}{ Item } & \multicolumn{2}{|c|}{ S1 } & \multicolumn{2}{|c|}{ S2 } & \multicolumn{2}{|c|}{ S3 } & \multirow[t]{2}{*}{ P1 } & \multirow[t]{2}{*}{ P2 } & \multirow{2}{*}{$\begin{array}{c}\text { Correlation } \\
\text { coefficient }\end{array}$} \\
\hline & Mean & SD & Mean & SD & Mean & $\mathrm{SD}$ & & & \\
\hline Age & 41.52 & 7.70 & 49.21 & 9.30 & 44.13 & 9.77 & 0.027 & 0.866 & 0.019 \\
\hline WC & 107.22 & 10.72 & 111.58 & 11.88 & 118.74 & 6.44 & $<0.001$ & 0.001 & 0.373 \\
\hline BMI & 33.00 & 4.61 & 33.76 & 4.10 & 36.29 & 4.16 & 0.009 & 0.001 & 0.356 \\
\hline ALT & 40.90 & 31.17 & 64.26 & 46.69 & 48.46 & 29.02 & 0.091 & 0.504 & 0.075 \\
\hline HDL & 42.35 & 8.97 & 48.57 & 33.04 & 44.86 & 17.19 & 0.618 & 0.492 & -0.077 \\
\hline LDL & 149.70 & 62.96 & 139.37 & 48.20 & 125.65 & 52.11 & 0.238 & 0.035 & -0.235 \\
\hline TGs & 170.91 & 89.68 & 203.68 & 99.29 & 313.87 & 63.30 & $<0.001$ & $<0.001$ & 0.500 \\
\hline
\end{tabular}

WC: waist circumference

FibroScan (Echosens ${ }^{\circledast}$ ) was mild (F0, F1) in $91.4 \%$ (57 patients F0, 17 patients $\mathrm{F} 1$ ), moderate (F2) in $0 \%$ and marked in $8.6 \%$ (F3 in 4 and F4 in 3 patients).

Correlation between the degree of steatosis by CAP and different parameters

The degree of steatosis was higher among females than males, with $39.1 \%$ of males and $60.9 \%$ of females having values corresponding to S1, $42.1 \%$ versus $57.9 \%$ having S2, and $38.5 \%$ versus $61.5 \%$ having S3 among males and females respectively. However, there was no significant difference $(\mathrm{P}=0.964)$.

By univariate analysis, among parameters shown in Table III, age, WC, BMI and higher TGs were significantly correlated with the degree of steatosis measured by CAP. While WC, BMI, LDL and TGs correlated with the mere presence of steatosis by CAP.

When multivariate regression analysis was conducted, all the independent risk factors showed non-significant correlations with the degree of steatosis on CAP. However, when using a stepwise approach, only the WC showed significance as an independent predictor of NAFLD (Table IV).

The different grades of brightness by US showed significant correlation with the degree of steatosis by CAP (P value

Table IV. Multivariate analysis of steatosis presence in relation to different factors

\begin{tabular}{lcccccc}
\hline Item & OR & $\begin{array}{c}\text { Standard } \\
\text { Error }\end{array}$ & Z & P & \multicolumn{2}{c}{$95 \%$ CI } \\
\hline Age & 0.98 & 0.12 & -0.16 & 0.87 & 0.77 & 1.24 \\
Sex & 26.41 & 92.17 & 0.94 & 0.35 & 0.03 & 24703.97 \\
Waist C & 5.025 & 2.01 & 0.15 & 0.997 & 0.88 & 1.09 \\
BMI & 24.76 & 48.88 & 1.63 & 0.10 & 0.52 & 1146.84 \\
WBC & 0.82 & 0.59 & -0.27 & 0.79 & 0.20 & 3.38 \\
AST & 1.28 & 0.29 & 1.08 & 0.28 & 0.82 & 2.01 \\
ALT & 1.03 & 0.18 & 0.14 & 0.89 & 0.73 & 1.45 \\
ALP & 1.08 & 0.05 & 1.55 & 0.12 & 0.98 & 1.19 \\
GGT & 0.90 & 0.10 & -0.96 & 0.34 & 0.73 & 1.11 \\
AA mutation & 0.26 & 0.52 & -0.67 & 0.50 & 0.01 & 13.02 \\
\hline \multicolumn{7}{c}{ Stepwise Multivariate analysis } \\
\hline WAIST C & OR: $1.655(1.327-2.063)$ & & P $<0.001$ \\
\hline
\end{tabular}

Cholesterol was set as low/high at a cutoff 200, TG were set as low/high at a cut of 150. (All patients without NASH had low cholesterol and low TG, so they could not be introduced in the multivariate analysis).
$<0.001$ ). Grade I brightness had 12 (S1), 7 (S2) and 3 (S3) patients according to CAP, grade II brightness had 9 (S1), 12 (S2) and 19 (S3) according to CAP and grade III brightness had 2 (S1), 0 (S2) and 17 (S3) patients according to CAP.

NNMT gene rs694539-variant among patients with NAFLD and its relation to CAP, FibroScan and clinical parameters

The comparison of rs694539-NNMT A and G alleles genotype distribution between NAFLD patients and controls showed that A allele was significantly higher in patients $(\mathrm{P}<0.001), \mathrm{G}$ allele was significantly higher in controls $(\mathrm{P}<0.001)$. The mutant AA-genotype was found in $33.3 \%$ of patients and $1.2 \%$ controls with high significance $(\mathrm{P}<0.001)$ and $21 \%$ of patients had the wild GG-genotype versus $64 \%$ controls with high significance $(\mathrm{P}<0.001)$ (Table V, Fig. 1).

The presence of the mutant AA-genotype showed a significant correlation with the degree of steatosis detected by CAP and a non-significant correlation with the degree of fibrosis (by FibroScan) (Table VI).

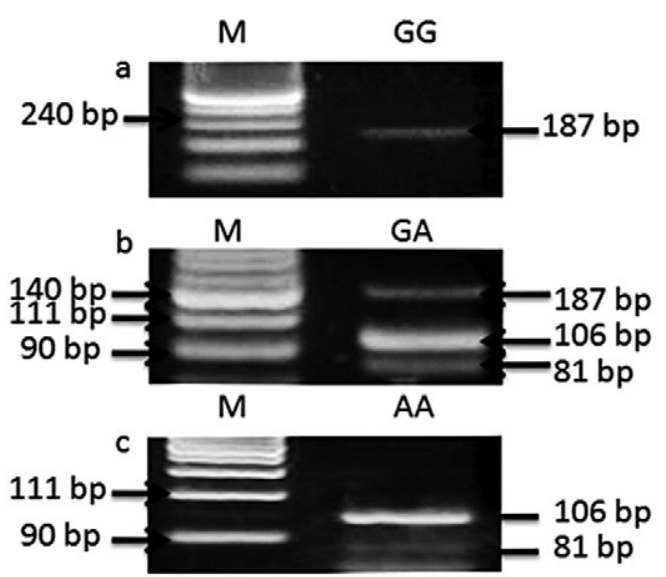

Fig. 1. The NNMT rs694539 genetic polymorphism using PCR-RFLP. The amplified $187 \mathrm{bp}$ fragment was cut with the restriction endonuclease NlaIII and analyzed on a $4 \%$ agarose gel electrophoresis. Lane $\mathrm{M}$ showing the marker (M). (a) lane GG showing the GG-genotype with one fragment of $187 \mathrm{bp}$; (b) lane GA showing the GA-genotype with three fragments of 187, 106, and 81 bp; and (c) lane AA showing the AA-genotype with two fragments of 106 and $81 \mathrm{bp}$. 
Table V. NNMT rs694539 variant among NAFLD patients versus controls and in obese versus nonobese individuals

\begin{tabular}{lcccccc}
\hline \multirow{2}{*}{$\begin{array}{l}\text { NNMT rs694539 } \\
\text { variant }\end{array}$} & \multicolumn{2}{c}{ Patients $(81)$} & \multicolumn{2}{c}{ Controls $(80)$} & P value & OR (95\%CI) \\
\cline { 2 - 7 } & No & $\%$ & No & $\%$ & & \\
\hline Wild GG & 17 & 21.0 & 51 & 63.8 & $<0.001$ & $0.151(0.075-0.305)$ \\
\hline GA & 37 & 45.7 & 28 & 35.0 & 0.167 & $1.562(0.828-2.945)$ \\
Mutant AA & 27 & 33.3 & 1 & 1.2 & $<0.001$ & $39.5(5.210-299.469)$ \\
G Allele & 71 & 43.8 & 130 & 81.2 & $<0.001$ & $0.180(0.109-0.298)$ \\
A Allele & 91 & 56.2 & 30 & 18.8 & $<0.001$ & $5.552(3.356-9.193)$ \\
\hline \multicolumn{7}{c}{ According to the BMI } \\
BMI & Obese $(64)$ & Non obese $(97)$ & P value & OR (95\%CI) \\
Wild GG & 14 & 21.9 & 54 & 55.7 & $<0.001$ & $0.223(0.109-0.456$ \\
GA & 30 & 46.9 & 35 & 36.1 & 0.172 & $1.563(0.822-2.972)$ \\
Mutant AA & 20 & 31.2 & 8 & 8.2 & $<0.001$ & $5.057(2.046-12.387)$ \\
G Allele & 58 & 45.3 & 143 & 73.7 & $<0.001$ & $0.296(0.184-0.474)$ \\
A Allele & 70 & 54.7 & 51 & 26.3 & $<0.001$ & $3.384(2.110-5.428)$ \\
\hline
\end{tabular}

Table VI. Correlation between NNMT rs694539 AA genotype and the degree of steatosis by CAP (S) and fibrosis by FibroScan (F)

\begin{tabular}{lccccc}
\hline \multirow{2}{*}{$\begin{array}{l}\text { FAP \& } \\
\text { Fibscan }\end{array}$} & \multicolumn{3}{c}{ rs694539variant of NNMT=AA } & \multirow{2}{*}{ P value } \\
\cline { 2 - 4 } & \multicolumn{3}{c}{ Yes } & No & \\
\cline { 2 - 5 } S1 & Count & $\%$ & Count & $\%$ & \\
S2 & 4 & 14.8 & 19 & 35.2 & \multirow{2}{*}{0.05} \\
S3 & 10 & 37.0 & 9 & 16.7 & \\
F0 & 13 & 48.2 & 26 & 48.1 & \\
F1 & 15 & 55.6 & 42 & 77.8 & \\
F3 & 9 & 33.3 & 8 & 14.8 & 0.135 \\
F4 & 2 & 7.4 & 2 & 3.7 & \\
\hline
\end{tabular}

The relation between the mutant AA-genotype of NNMTrs694539 and different clinical parameters showed that in patients with the mutant AA genotype, WC was $114.30 \pm 12.50$, BMI was $35.05 \pm 4.70$, TGs level was $248.63 \pm 94.50$ and ALT was $54.52 \pm 33.70$. While in patients without the mutant AA genotype, WC was $113.54 \pm 9.32$, BMI was $34.62 \pm 4.41$, TGs was $246.83 \pm 107.95$, and ALT was $47.77 \pm 35.86$. These correlations showed no statistical significance.

The mutant AA genotype and the A allele were significantly higher in obese vs. non-obese subjects $(P<0.001)$, on the contrary, the wild GG genotype and the $G$ allele were significantly higher in non-obese subjects $(\mathrm{P}<0.001)$ (Table V).

\section{DISCUSSION}

NALFD is considered the hepatic manifestation of obesity and MS. Progression from NALFD to NASH increases the risk of liver-related outcomes [11]. So, the knowledge of whether a patient has simple steatosis or NASH is important prognostically [12].

Several non-invasive modalities have demonstrated promise for aiding in the diagnostic approach of NASH. These include serum markers, both direct and indirect, clinical scoring systems, and novel imaging modalities, such as shear wave elastography.

In the last years, the genetic determinants of NAFLD are being unrevealed using genome-wide association studies (GWAS), which offer a powerful technique for discovering novel associations between SNPs and disease phenotypes. Newly-identified genetic risk variants could provide a useful tool for the clinical management and prognosis of obese patients with NAFLD [4].

The rs694539-variant of the NNMT gene is a SNP that is found to be significantly associated with hyperhomocysteinemia which causes steatosis [13]. Sazci et al. found that individuals with the GG-genotype of NNMT gene rs694539-variant appeared to have protection against $\mathrm{NASH}$, whereas persons with the AA-genotype showed significant increased risk for NASH, thereby reporting for the first time the rs694539-variant of NNMT gene as a genetic risk factor for developing NASH [5].

The BMI and WC were significantly higher in our NAFLD cases than in controls. Previous studies concluded that the prevalence of NAFLD increases with obesity, ranging between $34.2 \%$ and $60.9 \%$ as recently reported in a meta-analysis [1]. Other studies, however, concluded that NAFLD can occur in non-obese patients who are physically inactive $[14,15]$. In addition, some studies reported that WC served as a reliable predictor for the risk of NAFLD among obese patients [16].

In our study, the prevalence of DM and MS was significantly higher in NAFLD patients compared to controls. Many studies showed that the prevalence of NAFLD and NASH is higher among diabetics, with a prevalence of $22.51 \%$ and $43.63 \%$, respectively and considered NAFLD and NASH as the hepatic manifestation of MS, with an overall pooled prevalence of $42.54 \%$ and $70.65 \%$, respectively (1). Other studies also identified DM as an independent risk factor for the progression of NAFL [17].

In one study, the prevalence estimates of hyperlipidemia/ dyslipidemia among NAFLD and NASH obese patients were $69.16 \%$ and $72.13 \%$, respectively, and those of hypertriglyceridemia were $40.74 \%$ and $83.33 \%$ for NAFLD and 
NASH, respectively (1). Similarly, among our patients, elevated cholesterol, LDL-c and TG levels, as well as reduced HDL-c levels correlated to NAFLD presence. Furthermore, higher TGs and lower LDL-c correlated with steatosis degree detected by CAP.

Previous studies showed that, in up to nearly $60 \%$ of NAFLD obese patients with NASH, ALT could be normal $[18,19]$. Nevertheless, ALT can be increased in up to $53 \%$ of NAFLD patients without NASH $[18,20]$. Those studies thereby concluded that ALT level alone is not predictive of NASH or fibrosis level [20].

In our study, both ALT and AST levels were found to be significantly higher among NAFLD patients versus controls. Moreover, GGT was significantly higher among NAFLD patients as well. Pulzi et al. found that GGT $\geq 30 \mathrm{IU} / \mathrm{L}$ is an adequate predictive marker of NASH, others considered GGT as a marker of increased mortality [21]. On the other hand, Tahan et al. divided obese NAFLD patients into normal GGT and high GGT groups and found that differences in reference to histological steatosis grade and inflammation were not significant [22].

In comparison with abdominal US, in addition to being non-ionizing and inexpensive, CAP presents the added benefits of being machine-independent and non-subject to operator interpretation. Furthermore, CAP was shown to be efficient to offer quantification of hepatic steatosis from $10 \%$ and up. All this combined with the simultaneous assessment of liver fibrosis using liver stiffness makes CAP a more appealing novel method for assessing hepatic steatosis [23].

The degree of steatosis, in our study, correlated with the increase in age but not the gender difference. Previous studies identified age and male gender as independent risk factors for progression of steatosis $[24,25]$; the reason why the later finding could not be reproduced in our study could be due to the higher prevalence of females.

The increased BMI and WC and the worsening lipid profiles correlated with the degree of steatosis detected by CAP in our study. Previous studies demonstrated the same correlations in BMI and WC $[23,26]$ and serum TGs and LDL $[26,27]$.

SNPs of different genes were evaluated for prediction and differentiating simple steatosis from NASH. Our study showed consistent results to those demonstrated by Sazci et al. [5]. The mutant AA-genotype was found in $33.3 \%$ of NAFLD patients and in only $1.2 \%$ of controls, the wild GG was found in $21 \%$ of NAFLD patients versus $63.8 \%$ controls. Moreover, the mutant AA genotype and the A allele were significantly more frequent in obese subjects and on the contrary, the wild GG genotype and the $\mathrm{G}$ allele were significantly more frequent in non-obese subjects. From these results, we can conclude that the NNMT gene rs694539-variant mutant AA-genotype significantly correlates with the presence of NAFLD in obese Egyptians, while the wild GG-genotype appeared to offer protection against its development.

Moreover, the degree of steatosis detected by CAP appeared to significantly correlate with the presence of the mutant AAgenotype, where the percentage of patients with the mutant AA-genotype increased in correlation to the degree of steatosis.

Although the mutant AA genotype significantly correlated with steatosis presence and degree, it did not correlate with the degree of fibrosis (which is the major prognostic determinant in NAFLD). The NNMT gene (and its expressions) were not considered in this study and the previous one study as noninvasive fibrosis markers. Nevertheless, marked steatosis degrees have been proved by this study and other studies that they will mostly and eventually progress (with a second hit) to inflammation and fibrosis.

Therefore, the major outcome from the clinical point of view elaborates that the mutant AA genotype could be a good prognostic marker as it can diagnose marked steatosis (higher susceptibility of NASH progression) and most importantly can predict clinically significant fibrosis and its complications years before its development.

Further studies should be performed to combine the NNMT gene with the other major genetic risk factors for NAFLD, e.g. PNPLA3, TM6SF2, MBOAT7 and GCKR variants.

Also, studies with larger sample size are required and results should be replicated in an independent cohort with a different ethnicity to ascertain this study conclusions.

\section{CONCLUSIONS}

As found in our study, polymorphisms in rs694539-NNMT gene (mutant AA-genotype) could be a genetic risk factor for developing NAFLD and NASH (indicating susceptibility for progression and complications). Individuals with wild type (GG-genotype) are at less risk from NAFLD development. CAP and FibroScan efficiently diagnosed steatosis and fibrosis, respectively. The degree of steatosis by CAP correlated significantly with age, BMI, WC, and high TG levels.

\section{Conflicts of interest: None declared.}

Authors' contributions: E.M.H., R.A.A.A. and A.Y.: design of the work and supervision; R.A.A.A., H.D., S.D., and A.E.: data collection; D.S.: laboratory work; S.D.: idea and work design, writing and reviewing the paper; H.D.: interviewing the patients, writing the paper; A.E.: revision of the paper; M.A.: revision, editing and then submission of the manuscript; A.Y.: supervision of the work.

\section{REFERENCES}

1. Younossi ZM, Koenig AB, Abdelatif D, Fazel Y, Henry L, Wymer M. Global epidemiology of nonalcoholic fatty liver disease-Meta-analytic assessment of prevalence, incidence, and outcomes. Hepatology 2016;64:73-84. doi:10.1002/hep.28431

2. Chalasani N, Younossi Z, Lavine JE, et al. The diagnosis and management of non-alcoholic fatty liver disease: practice Guideline by the American Association for the Study of Liver Diseases, American College of Gastroenterology, and the American Gastroenterological Association. Hepatology 2012;55:2005-2023. doi:10.1002/hep.25762

3. Hirschhorn JN, Gajdos ZK. Genome-wide association studies: results from the first few years and potential implications for clinical medicine. Ann Rev Med 2011;62:11-24. doi:10.1146/annurev.med.091708.162036

4. Dongiovanni P, Romeo S, Valenti L. Genetic Factors in the Pathogenesis of Nonalcoholic Fatty Liver and Steatohepatitis. BioMed Res Int 2015;2015:460190. doi:10.1155/2015/460190

5. Sazci A, Ozel MD, Ergul E, Aygun C. Association of nicotinamide$\mathrm{N}$-methyltransferase gene rs694539 variant with patients with 
nonalcoholic steatohepatitis. Genet Test Mol Biomarkers 2013;17:849 853. doi:10.1089/gtmb.2013.0309

6. Festi D, Schiumerini R, Marasco G, Scaioli E, Pasqui F, Colecchia A. Non-invasive diagnostic approach to non-alcoholic fatty liver disease: current evidence and future perspectives. Expert Rev Gastroenterol Hepatol 2015;9:1039-1053. doi:10.1586/17474124.2015.1049155

7. Brunt EM, Kleiner DE, Wilson LA, Belt P, Neuschwander-Tetri BA; NASH Clinical Research Network (CRN). Nonalcoholic fatty liver disease (NAFLD) activity score and the histopathologic diagnosis in NAFLD: distinct clinicopathologic meanings. Hepatology 2011;53:810820. doi:10.1002/hep. 24127

8. de Ledinghen V, Vergniol J. Transient elastography (FibroScan). Gastroenterol Clin Biol 2008;32(6 Suppl 1):58-67. doi:10.1016/S0399. 8320(08)73994-0

9. de Ledinghen V, Vergniol J, Capdepont M, et al. Controlled attenuation parameter (CAP) for the diagnosis of steatosis: a prospective study of 5323 examinations. J Hepatol 2014;60:1026-1031. doi:10.1016/j. jhep.2013.12.018

10. Rodriguez S, Gaunt TR, Day IN. Hardy-Weinberg equilibrium testing of biological ascertainment for Mendelian randomization studies. Am J Epidemiol 2009;169:505-514. doi:10.1093/aje/kwn359

11. Angulo P, Kleiner DE, Dam-Larsen S, et al. Liver Fibrosis, but No Other Histologic Features, Is Associated With Long-term Outcomes of Patients With Nonalcoholic Fatty Liver Disease. Gastroenterology 2015;149:389-397.e10. doi:10.1053/j.gastro.2015.04.043

12. Dyson JK, Anstee QM, McPherson S. Non-alcoholic fatty liver disease: a practical approach to diagnosis and staging. Frontline Gastroenterol 2014;5:211-218. doi:10.1136/flgastro-2013-100403

13. Bromberg A, Lerer E, Udawela $M$, et al. Nicotinamide-N methyltransferase (NNMT) in schizophrenia: genetic association and decreased frontal cortex mRNA levels. The Int J Neuropsychopharmacol 2012;15:727-737. doi:10.1017/S1461145711001179

14. Caldwell S, Argo C. Non-Alcoholic Fatty Liver Disease and Nutrition. In: Dooley JS, Lok AS, Burroughs AK, Heathcote EJ. (Eds.). Sherlock's Diseases of the Liver and Biliary System. 12th ed. Blackwell Publishing Ltd., 2011:546-567. doi:10.1002/9781444341294

15. Arvaniti VA, Thomopoulos KC, Tsamandas A, et al. Serum adiponectin levels in different types of non alcoholic liver disease. Correlation with steatosis, necroinflammation and fibrosis. Acta Gastroenterol Belg 2008;71:355-360.

16. Clemente AP, Netto BD, de Carvalho-Ferreira JP, et al. Waist circumference as a marker for screening nonalcoholic fatty liver disease in obese adolescents. Rev Paul Pediatr 2016;34:47-55. doi:10.1016/j. rppede.2015.10.004

17. Hossain N, Afendy A, Stepanova M, et al. Independent predictors of fibrosis in patients with nonalcoholic fatty liver disease. Clin Gastroenterol Hepatol 2009;7:1224-1229. doi:10.1016/j.cgh.2009.06.007

18. Fracanzani AL, Valenti L, Bugianesi E, et al. Risk of severe liver disease in nonalcoholic fatty liver disease with normal aminotransferase levels: a role for insulin resistance and diabetes. Hepatology 2008;48:792-798. doi:10.1002/hep.22429

19. Verma S, Jensen D, Hart J, Mohanty SR. Predictive value of ALT levels for non-alcoholic steatohepatitis (NASH) and advanced fibrosis in non-alcoholic fatty liver disease (NAFLD). Liver Int 2013;33:1398-1405 doi:10.1111/liv.12226

20. Torres DM, Harrison SA. NAFLD: Predictive value of ALT levels for NASH and advanced fibrosis. Nat Rev Gastroenterol Hepatol 2013;10:510-511. doi:10.1038/nrgastro.2013.138

21. Pulzi FB, Cisternas R, Melo MR, Ribeiro CM, Malheiros CA, Salles JE. New clinical score to diagnose nonalcoholic steatohepatitis in obese patients. Diabetol Metab Syndr 2011;3:3. doi:10.1186/1758 5996-3-3

22. Tahan V, Canbakan B, Balci H, et al. Serum gamma-glutamyltranspeptidase distinguishes non-alcoholic fatty liver disease at high risk. Hepatogastroenterology 2008;55:1433-1438.

23. de Ledinghen V, Vergniol J, Foucher J, Merrouche W, le Bail B. Noninvasive diagnosis of liver steatosis using controlled attenuation parameter (CAP) and transient elastography. Liver Int 2012;32:911-918. doi:10.1111/j.1478-3231.2012.02820.x

24. Noureddin M, Yates KP, Vaughn IA, et al. Clinical and histological determinants of nonalcoholic steatohepatitis and advanced fibrosis in elderly patients. Hepatology 2013;58:1644-1654. doi:10.1002/ hep. 26465

25. Torres DM, Williams CD, Harrison SA. Features, diagnosis, and treatment of nonalcoholic fatty liver disease. Clin Gastroenterol Hepatol 2012;10:837-858. doi:10.1016/j.cgh.2012.03.011

26. Petta S, Amato MC, Di Marco V, et al. Visceral adiposity index is associated with significant fibrosis in patients with non-alcoholic fatty liver disease. Aliment Pharmacol Ther 2012;35:238-247. doi:10.1111/ j.1365-2036.2011.04929.x

27. Kashyap SR, Diab DL, Baker AR, et al. Triglyceride levels and not adipokine concentrations are closely related to severity of nonalcoholic fatty liver disease in an obesity surgery cohort. Obesity (Silver Spring) 2009;17:1696-1701. doi:10.1038/oby.2009.89 